\title{
LA RESEMANTIZACIÓN DEL BRUTO DE DAVID DURANTE LA REVOLUCIÓN FRANCESA: DE PROPAGANDA MONÁRQUICA A OBRA REVOLUCIONARIA*
}

\author{
THE RESEMANTIZATION OF DAVID'S BRUTUS \\ DURING FRENCH REVOLUTION: \\ FROM MONARCHIC PROPAGANDA \\ TO A REVOLUTIONARY ARTWORK
}

\author{
ELENA BELLIDO PÉREZ \\ Universidad de Sevilla \\ https://orcid.org/0000-0002-3107-5481
}

Recibido: 20/09/2020 Evaluado: 30/11/2020 Aprobado: 09/12/2020

RESUMEN: Jacques-Louis David se involucró activamente con todos los poderes de la Francia de finales del siglo XVIII: pintó para el rey Luis XVI, para los jacobinos y para el emperador Napoleón. Sus contradicciones ideológicas tienen su razón de ser en la ambición profesional del artista, máximo representante del neoclasicismo, cuyo objetivo de acabar con la Academia lo llevó a las más altas esferas de la política revolucionaria. Del estudio de su obra Los lictores devuelven a Bruto los cadáveres de sus hijos se desprende tanto el viraje ideológico de David como el incipiente rol del artista independiente del cambio de siglo.

"Este trabajo se ha realizado en el contexto del grupo de investigación IDECo (Grupo de Investigación en Comunicación Política, Ideología y Propaganda, SEJ-539). 
Palabras clave: arte, propaganda, Revolución Francesa, Jacques-Louis David, Bruto.

ABSTRACT: Jacques-Louis David was actively involved with all the powers of late 18th century in France: he painted for King Louis XVI, for the Jacobins and for the Emperor Napoleon. His ideological contradictions have their raison d'etre in the professional ambition of the artist, who was the highest figure of Neoclassicism, whose objective of ending with the Academy led him to the highest spheres of revolutionary politics. The study of his work Lictors Returning to Brutus the Bodies of his Sons reveals both David's ideological turn and the incipient role of the independent artist belonged to the turn of the century.

Keywords: art, propaganda, French Revolution, Jacques-Louis David, Brutus.

\section{INTRODUCCIÓN: DAVID EN SU CONTEXTO}

El trabajo de Jacques-Louis David como pintor se sitúa en la Francia del cambio de siglo XVIII al XIX, años en los que el país estaba experimentando una serie de acontecimientos que cambiarían el curso de la historia occidental. David nació el 30 de agosto de 1748 en París y murió en Bruselas, enfermo del corazón, 77 años más tarde. Esta larga vida fue la que le permitió involucrarse en todos los cambios históricos que pudo presenciar. Como comenta Schnapper, «he lived through the Ancien Régime, the Revolution, the Consulate, the Empire and the Restoration», y aunque esto también se aplique a otros artistas coetáneos, «none was so closely involved in the events of his time». ${ }^{1} \mathrm{Y}$ es que David no solo ilustró los tiempos convulsos de la Francia revolucionaria, sino que desarrolló un trabajo dialogante con su época, adoptando un papel activo en los conflictos y en las decisiones políticas, y trasladando al arte y a la cultura europea, a través de su estilo neoclásico, un patriotismo que pasó a ocupar un lugar predominante en el Romanticismo posterior.

En su rol político, David fue un miembro de la Convención, un regicida, un íntimo de Maximilien Robespierre y el esteta de Napoleón Bonaparte, variando en tal grado en su posicionamiento ideológico, que los autores que han estudiado al artista no encontraban dificultades en interpretar la obra

\footnotetext{
1. Antoine Schnapper: David, Alpine Fine Arts Collection, Nueva York, 1982, p. 7.
} 
del pintor en función de su propio convencimiento político. ${ }^{2}$ No obstante, tal y como apunta Lee, en la motivación artística de David son clave su propia competitividad y la búsqueda de fama y reconocimiento. ${ }^{3}$ Así, Lee acaba afirmando que "perhaps the characteristic of David that emerges most clearly is his combination of powerful commitment and extreme pragmatism». ${ }^{4}$ Nada más lejos de la realidad: aunque el curso de la historia se encargue de encajar razonadamente el posicionamiento político adoptado por David en cada momento, el hecho de que su compromiso destacara por igual en todas las etapas lleva a señalar como su carta motivacional un pragmatismo que va desde la supervivencia artística hasta la pura ambición profesional.

Sin embargo, existe un nexo de unión entre la mayoría de obras de David y su puesta al servicio de diferentes causas y entidades de poder: este es el patriotismo. Tal concepto viene dado por el contexto cultural en el que se desarrolló su obra. Según Brookner, aunque no haya evidencias de que David fuese lector de Rousseau, La Font de Saint-Yenne, Johann-Joachim Wincklemann o Denis Diderot, su trabajo se puede enmarcar en los ideales de dichos autores, populares en su tiempo, dada su «vindication of the century's nostalgia for nature and for nobility, for moral worth and the aesthetic sublime, for civic joy and for political devotion, and in the purest sense of liberty, equally, and fraternity». ${ }^{5}$

Por otro lado, del contexto artístico de David se han de destacar los Salones y la Academia, instituciones ambas dependientes de la Corona antes de la Revolución Francesa. Los Salones de París, celebrados en el Palacio del Louvre, se habían consagrado como eventos bienales desde $1737,{ }^{6}$ y jugarían un rol fundamental en el reconocimiento posterior de la obra de David. En ellos, donde acudían personas de todas las clases sociales, se fraguó la figura del público como ente determinante para la comunicación artística ${ }^{7}$ y, por consiguiente, para el comercio de arte que se consolidaría tras la emancipación de los artistas de las antiguas instituciones de poder. Poco a poco, las élites que intermediaban en el contenido y la forma de las obras quedaron reducidas: en los Salones, «the ordinary man or woman was encouraged to rehearse before works of art the kinds of pleasure and discrimination that once had been the exclusive prerogative of the patron and his intimates». ${ }^{8}$

2. Ibidem

3. Simon LeE: David, Phaidon, Londres, 1999, p. 6.

4. Ibidem

5. Anita Brookner: Jacques-Louis David, Purnell, Paulton, 1980, p. 23.

6. Thomas Crow: Painters and Public Life in Eighteenth Century Paris, Yale University Press, New Haven, 2000, p. 1

7. Ibidem.

8. Ibidem, pp. 2-3. 
En cuanto a la Academia, esta era la única vía posible para acceder a los Salones y para, en definitiva, tener un futuro artístico. En la época de David, la Academia estaba controlada por el conde D'Angiviller, al mando de la Direction des Bâtiments du Roi. Cuando este relevó al marqués de Marigny, insufló un cambio fundamental: propició el género de la pintura de historia como medio transmisor de la virtud heroica. «He recognized the propaganda value of history painting», comenta Boime, «and wished not only to convey a concern for the political and moral virtues extolled by the rising bourgeoisie but to restore the nationalistic sentiment so badly damaged in the wake of the Seven Years War». ${ }^{9}$ La novedad del programa de pintura de historia propuesto por D'Angiviller para el rey era, tal y como indica Honour, que las virtudes que pretendía transmitir con él eran abstractas y no se asociaban nunca a la monarquía de manera directa, como era costumbre hasta ese momento. ${ }^{10}$ Este hecho, sin haberlo previsto, configuraría la estética revolucionaria posterior y haría de David un artista destacado en su independencia ideológica.

Protegido por Joseph-Marie Vien, David ingresó en la Academia a los 18 años. A partir de entonces, los esfuerzos del pintor se concentraron en el Premio de Roma, un prestigioso galardón que becaba al ganador durante tres años en la Academia Francesa en Roma, donde los estudiantes copiaban a los maestros clásicos como Rafael o Tiziano. David intentaría hacerse con él en tres ocasiones fallidas antes de conseguirlo en $1774 .{ }^{11}$ De espíritu considerablemente ambicioso, estos rechazos le provocaron incluso un intento de suicidio, pero, sobre todo, un sentimiento encontrado con la institución oficial de la Academia. No obstante, David pudo disfrutar posteriormente del favor oficial realizando encargos como El juramento de los Horacios (1784) para el rey o La muerte de Sócrates (1787) para el concejal Charles-Michel Trudaine de la Sablière.

\section{LA CREACIÓN DE BRUTO}

El éxito de los Horacios en el Salón de 1785 hizo que David recibiese otro encargo de la Corona, materializado en Los lictores devuelven a Bruto los cadáveres de sus hijos, cuyo nombre completo es Brutus, premier Consul, de retour en sa maison, après avoir condamné ses deux fils, qui sétoient conspiré contre la liberté Romaine, des Licteurs rapportent leurs corps pour qu'on leur

9. Albert Boime: «Marmontel's Belisaire and the pre-revolutionary progressivism of David», en Art History, 3(1), 1980, pp. 81-101.

10. Hugh Honour: Neo-Classicism, Penguin Books, Victoria, 1968, p. 82.

11. Antoine Schnapper: David, 1982, pp. 22-25. 
donne la sépulture (figura 1). Paradójicamente, esta obra, concebida originalmente para servir como propaganda para la Corona, se convertiría en la carta de presentación del posterior republicanismo de David.

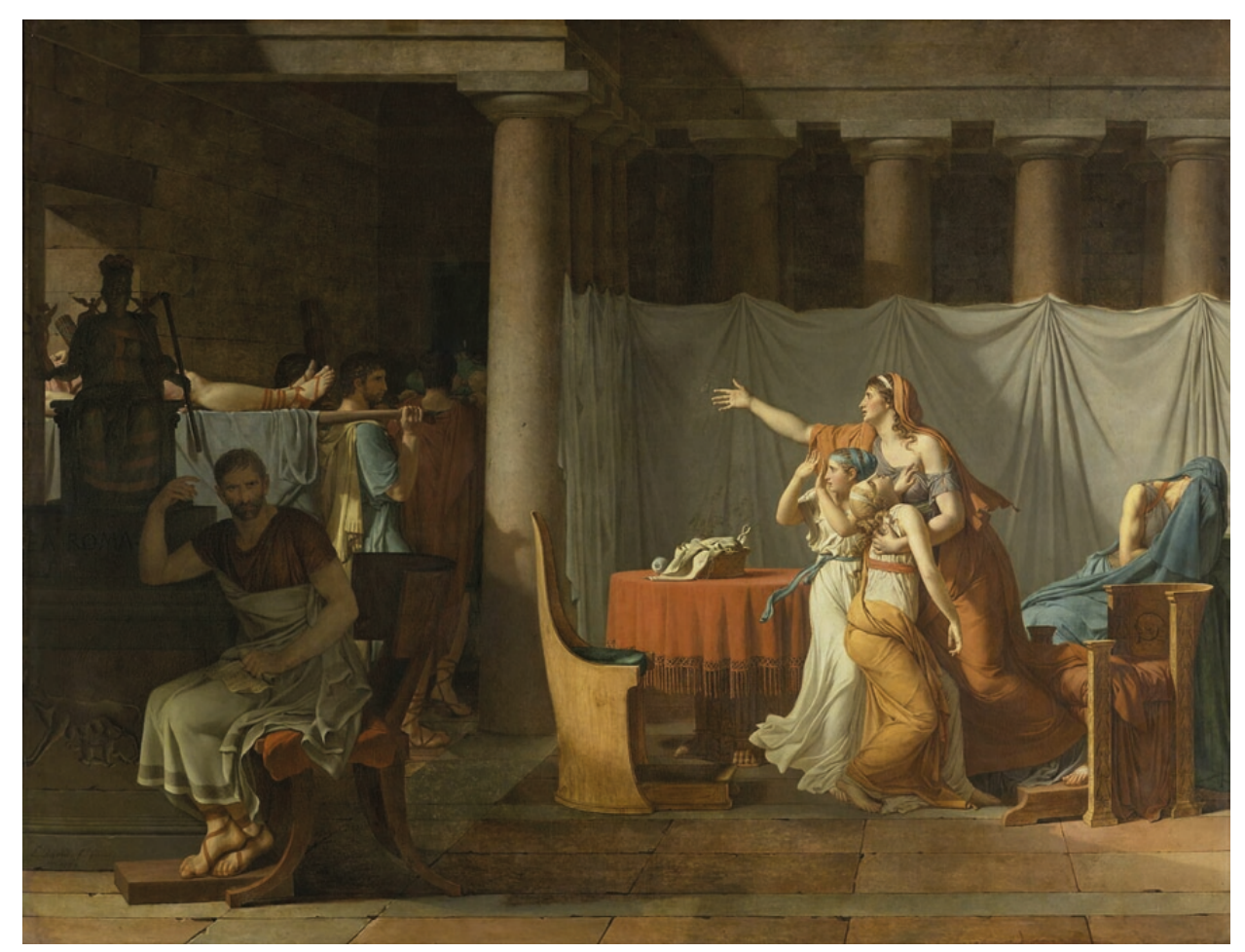

Figura 1: Los lictores devuelven a Bruto los cadáveres de sus hijos (Jacques-Louis David, 1789). Museo del Louvre, París, 323 x 422 cm

Dos años después de la exposición de los Horacios, el conde D'Angiviller le encargó a David una pintura de historia para la monarquía, dándole libertad en la elección del tema. ${ }^{12}$ Según Herbert, David propuso dos temas entonces: el general republicano Cayo Marcio Coriolano en el momento en que es persuadido por su familia para que no busque venganza sobre Roma, y la partida hacia la guerra del cónsul Marco Atilio Régulo, aceptando D’Angiviller el primer tema. ${ }^{13}$ Coriolano era un general conservador que se había ganado el respeto de la aristocracia y el desencanto del pueblo al no tomar medidas

12. Antonio Pinelli, David, 5 continents, Milán, 2004, p. 18.

13. Robert L. Herbert, David Brutus, Allen Lane, Londres, 1972, p. 18. 
contra la hambruna que padecía. ${ }^{14}$ Sin embargo, David cambió el tema por motivos de los cuales no queda constancia. Sí se conoce que el pintor no llegó a informar sobre este cambio: todavía en 1789, la correspondencia demuestra que la Casa Real entendía que David trabajaba en su Coriolano, una historia que, por otra parte, favorecía su interpretación monárquica. ${ }^{15}$

En estos años no existía todavía ningún documento que constatase el posicionamiento ideológico de David. De hecho, las interpretaciones republicanas de sus cuadros vinieron justo después de la exposición de Bruto en el Salón de 1789; esto es, justo después del estallido de la Revolución Francesa. $\mathrm{Si}$ bien es cierto que en ocasiones parecía desafiar a las instituciones oficiales de la Academia y la monarquía entregando tarde sus trabajos o cambiando las proporciones y temáticas de los encargos ${ }^{16}$ la realidad, a efectos prácticos, es que David seguía siendo un pintor academicista que recibía encargos reales por parte de D'Angiviller y los exponía en los salones. Estos actos de rebeldía se consideraban en su momento como la necesidad que tenía el artista de crear un efecto sorpresa en torno a su trabajo, pero no como la negación del poder real. ${ }^{17}$ Por consiguiente, en 1789 , los críticos de la época aún no comentaban que David estuviese poniendo en cuestión ni la Corona ni la Academia como organismo oficial regulador del arte, sino todo lo contrario: el artista trabajaba y se beneficiaba de ambos.

Así pues, desconociendo la existencia de una motivación ideológica en el cambio de temática del encargo, David representó finalmente una escena íntima de la historia de Bruto. ${ }^{18}$ Lucio Junio Bruto era el sobrino de Lucio Tarquinio el Soberbio, séptimo y último rey de Roma. Este era un tirano que, tras matar a su esposa para poder casarse con la hija del rey, asesinó también a su suegro para poder usurparle el trono. Tarquinio condenó a muerte a varios senadores, así como a la mayoría de la familia de su sobrino Bruto. La caída del déspota se inicia cuando su hijo Sexto Tarquinio violó a la patricia Lucrecia, la cual acabó suicidándose frente a su esposo, su padre y frente a Bruto. Este último, acabó jurando sobre el cadáver de Lucrecia que acabaría con la monarquía. Tras una exitosa batalla liderada por Bruto, los Tarquinios se exiliaron y Bruto se convirtió en cónsul junto con el que fuera esposo de Lucrecia, siendo así uno de los fundadores de la República. Para la desgracia de Bruto, sus dos hijos Tito y Tiberio estuvieron más tarde envueltos en una conspiración para la restauración de la monarquía, lo cual obligó a Bruto a ordenar la ejecución de ambos.

\footnotetext{
14. Ibidem, pp. 54-55.

15. Ibidem, p. 18.

16. Ibidem, p. 61.

17. Ibidem, p. 62.

18. Ibidem, pp. 16-17.
} 
Según Schnapper, David pudo cambiar el tema motivado por la tragedia Brutus de Voltaire, la cual, inequívocamente anti-absolutista, había sido representada una sola vez en $1786 .{ }^{19}$ Fuese la tragedia de Voltaire la razón o no, cuando David se decidió por Bruto para su encargo real, pretendió en un primer momento continuar la tradición pictórica de la historia del cónsul representando su juramento de venganza sobre el cuerpo de Lucrecia. ${ }^{20}$ Esta escena, junto con la condena a muerte de sus hijos, ya estaba comúnmente aceptada en la pintura como ejemplo de virtud heroica, habiendo sido propuesta por Diderot y La Font de Saint-Venne. ${ }^{21}$ En el año anterior, por ejemplo, el austríaco Friedrich Heinrich Füger y el francés Guillaume Guillon Lethière trabajaron sobre la misma temática. En definitiva, siempre se trataba de un héroe defendiendo su patria hasta las últimas consecuencias. No obstante, en 1788 David ya había decidido mostrar una escena nunca vista antes: la tensión de Bruto cuando recibe los cadáveres de los hijos que, llevado por el deber patriótico, había mandado asesinar. De este modo, la virtud heroica quedaba retratada en sus más crudas consecuencias, reflejada en el tormento interno del protagonista. Así, a través de una carta que dirigió a su alumno Wicar el 14 de junio de 1789, el propio David ratificó su voluntad de introducir este nuevo elemento en el imaginario cultural:

Estoy haciendo una pintura de invención propia. Bruto, hombre y padre, se ha privado de sus hijos y ha regresado a su hogar, donde le traen los cuerpos de sus dos hijos para enterrarlos. Es interrumpido en su angustia, al pie de la estatua de Roma, por los gritos de su esposa, el miedo y el desmayo de su hija mayor. La descripción es hermosa, pero en cuanto a la pintura, no me atrevo a decir nada todavía. Parece, para no mentirte, que están contentos con la composición. ${ }^{22}$

Tal y como se puede observar, no hay nada en esta descripción que implique un compromiso político por parte de David, ya que no menciona ni siquiera la causa republicana que motivaba al héroe a tomar esta decisión. David, por tanto, se mantenía aséptico con sus alumnos, y comentaba su obra desde un punto de vista exclusivamente iconográfico.

Por lo tanto, los motivos de creación de esta obra se pueden resumir del siguiente modo:

19. Antoine Schnapper, David, p. 92.

20. Ibidem, pp. 18-19.

21. Anita Brookner, Jacques-Louis ..., p. 24; Stefan Germer y Hubertus Kohle: «From the theatrical to the aesthetic hero: on the privatization of the idea of virtue in David's Brutus and Sabines», en Art History, 9(2), 1986, p. 171; Simon LeE: David, 1999, p. 122; Durning Lawrence Ettlinger: «Jacques Louis David and Roman Virtue», en Journal of the Royal Society of Arts, 115(5126), 1967, p. 117.

22. Traducción de la autora (en adelante, TdA) de David citado en Robert L. Herbert, David..., pp. 123-124. 
- En primer lugar, los motivos originales son externos: David emprende la creación de este cuadro porque la Corona le había encargado una pintura de historia. Es, por tanto, una obra de propaganda, aunque con un marco de libertad creativa bastante amplio.

- En segundo lugar, David debe decidir personalmente el tema y acaba desestimando el Coriolano acordado, de trazas conservadoras, para abordar la historia de Bruto, quizá motivado por la tragedia anti-monárquica de Voltaire.

- Y, en tercer lugar, David debe tomar de nuevo otra decisión: qué escena de Bruto representar. En este caso, la motivación puede derivarse de sus propias inquietudes estéticas y creativas, puesto que decide romper con la tradición histórica y mostrar un momento íntimo y familiar quebrado por el deber patriótico de Bruto.

Así pues, aunque el origen del Bruto sea un encargo monárquico, por la serie de decisiones que tuvo que tomar David en torno a su obra se aprecia un amplio espectro de posibilidades creativas en las que el pintor podía, además de innovar estéticamente, arriesgar ciertas interpretaciones ideológicas. Por ello, lo que sí puede afirmarse es que David era consciente en cierto modo de lo que su Bruto implicaría. Tal y como Schnapper comenta, David eligió una escena en la que se ponía antes de relieve el patriotismo de Bruto que su republicanismo: «What the painter exalts is the strength of mind, the stoicism, in the pure tradition of 'events illustrating virtues' such as painters had been producing in increasing numbers during the last fifteen years in the king's service»; ${ }^{23}$ aunque, por otro lado, «David must nevertheless have been aware of the political implications of the choice of such a subject in $1788 »{ }^{24}$ Ciertamente, Schnapper coincide con Boime cuando este último comenta que es imposible que David «no tuviera en cuenta las numerosas implicaciones de su tema con respecto a los acontecimientos que se estaban desarrollando», puesto que con la exposición del Bruto «su posición progresista coincidió con la de aquellos que asumieron el poder». ${ }^{25}$

23. Antoine Schnapper, David, p. 92.

24. Ibidem, p. 92.

25. Albert Boime: Historia social del arte moderno. 1. El arte en la época de la Revolución, 17501800, Alianza Forma, Madrid, 1994 (1987), pp. 425 y 436. 


\section{LA EXPOSICIÓN DE BRUTO EN EL SALÓN DE 1789}

\section{Controversias iniciales}

El año de 1789 fue un año determinante para Francia y, por extensión, para Europa. Desde 1781, los precios de la agricultura eran demasiado bajos, lo cual, junto con la sequía de 1785 y las grandes hambrunas de 1785 y $1788,{ }^{26}$ hizo que las revueltas populares no dejaran de sucederse. Los campesinos se encontraban quemando castillos a las afueras de París, destrozando vallas y matando a miembros de la nobleza ${ }^{27}$ antes de que ocurriese la toma de la Bastilla el 14 de julio de 1789, gracias a su vez a soldados descontentos que facilitaron el asalto. Los disturbios poco a poco dieron paso a una revolución, liderada por una burguesía descontenta y unos parlamentarios que habían sido destituidos de su cargo. La Asamblea Nacional se había autoproclamado como tal el 17 de junio de 1789 a propuesta de Sieyès. El 23 de junio, tres días después del juramento en el patio de juego de pelota, Luis XVI presidió una sesión conjunta donde adoptó una actitud desafiante contra la recién proclamada Asamblea y ordenó su disolución. ${ }^{28} \mathrm{Sin}$ embargo, acabó cediendo ante la Asamblea para prevenir más altercados. En los días sucesivos, la mayoría del clero y un grupo de nobles liderados por el duque de Orleans, primo del rey, pasaron a formar parte de la Asamblea Nacional. ${ }^{29}$ Finalmente, el 9 de julio se proclamó la Asamblea Nacional Constituyente y, tras la toma de la Bastilla y las tensiones entre la burguesía armada y el ejército del rey, ${ }^{30}$ se celebró una extensa reunión el 4 de agosto en la que se dijo de Luis XVI que sería el regenerador de la libertad francesa. ${ }^{31}$ Este hecho, en principio, marcaría el nuevo comienzo de Francia como monarquía constitucional. Así pues, la tarea de la Asamblea Nacional Constituyente era trabajar en una constitución donde se reconociesen los derechos del hombre, y se eliminasen la tortura judicial, los impuestos feudales y los privilegios fiscales; no obstante, no pidieron la abolición de la monarquía. ${ }^{32}$ Así, el 26 de agosto

26. James Llewelyn White: The origins of Modern Europe, Horizon Press Publishers, Nueva York, 1965 , p. 376.

27. Ibidem, p. 378.

28. Fernando Prieto: La Revolución Francesa, Itsmo Madrid, 1989, p. 19.

29. Ibidem, p. 19.

30. Cuando la Asamblea sospechó que el rey estaba reuniendo su ejército alrededor de Versalles para derrocarles, comenzó a armar a la burguesía, dando como resultado la toma de la Bastilla. Esta milicia burguesa en París pasó a llamarse la Guardia Nacional (al frente de la cual se situaba Lafayette) y, así la situación, algunos ministros le aconsejaron al rey que huyese. Él decidió quedarse, pero su hermano, el conde d'Artois y futuro Carlos X, sí abandonó París, al igual que muchos nobles que emigraron al extranjero (Fernando Prieto: La Revolución ..., p. 20).

31. Ibidem, pp. 20-21.

32. James Llewelyn White: The origins of Modern ..., p. 377. 
de 1789, un día después de la apertura del Salón de la Academia, se presenta como precedente constitucional la Declaración de los Derechos del Hombre $y$ del Ciudadano, con la que termina el sistema feudal. La Constitución no estaría terminada hasta pasados dos años: el 3 de septiembre de 1791.

El Salón de 1789 se organizó, como todos los anteriores, bajo el auspicio de la monarquía, completando así el destino para el que el Bruto fue concebido. La portada del libreto del Salón de 1789 patentaba la iniciativa del rey respecto a la exposición:

EXPlication des Peintures, SCulpture, et GRAVUres, De messieurs de L'ACADEMIE ROYALE, dont l'Exposition a été ordonnée, suivant l'intention de SA MAJESTÉ, par M. LE COMTE DE LA BILlARDRIE D'ANGIVILler, Conseiller du Roi en ses Conseils, Mesíre-de-Camp de Cavalerie, Chevalier de l'Ordre Royal \& Militaire de S. Louis, Commandeur de l'Ordre de S. Lazare, Gouverneur de Rambouillet, Directeur \& Ordonnateur-Général de Bâtiments de Sa Majesté, Jardins, Arts, Académies \& Manufactures Royales; de l’Acadèmie Royale des Sciences. ${ }^{33}$

El contenido de todo el Salón debía redundar, por tanto, en el beneficio de la monarquía. Por naturaleza, al tratarse de una obra de propaganda, la institución de poder que realiza el encargo debe estar presente desde la propia concepción de la misma. No obstante, en Los lictores devuelven a Brutos los cadáveres de sus hijos hallamos, diversas capas de lectura obtenidas al estudiar su contexto de creación. Concretamente, existen dos sentidos principales:

1. En primer lugar, Bruto solo sería un héroe patriótico utilizado por la Casa Real francesa para transmitirle al público la virtud heroica de servir a un país. Este es el sentido que la monarquía había pretendido con su encargo y que estaba presente, por otro lado, en toda la pintura de historia que desde 1774 el conde D'Angiviller estaba promoviendo para el rey. Esta sería, por tanto, la interpretación que la monarquía buscaba para el Bruto, facilitada por el contexto expositivo.

2. En segundo lugar, la monarquía seguiría estando presente en la obra de David, pero en forma de negación: es la necesidad de Bruto de acabar con una monarquía tiránica (y no la necesidad de defender de manera abstracta la nación) la que le obliga a anteponer sus deberes como patriota a sus deberes como padre. De este modo, Bruto le estaría mostrando al público francés un ejemplo de virtud patriótica extensible a todos los ciudadanos, y no aplicable, como se pretendía en el punto

33. Sin Autor: Explication des Peintures, Sculptures, et Gravures, de Messieurs de l'Académie Royale, Imprimerie des Bâtimens du Roi, París, 1789. 
anterior, a los valores de la Casa Real. Este es el sentido crítico que perviviría en la obra, ${ }^{34}$ olvidándose así su concepción original como propaganda monárquica.

Ciertamente, el Bruto en el contexto del Salón de 1789 suscitaría una preocupación explícita en el poder real que, en aquellos momentos, era el blanco de todas las revueltas populares. Las virtudes ejemplares en el arte (propuestas por D’Angiviller) se estaban trabajando al límite de lo que la monarquía podía permitir.

El cuadro de David no se expuso el día de la inauguración, ${ }^{35}$ sino el 12 de septiembre ${ }^{36}$ siendo el retraso ya habitual en el artista. El conde D’Angiviller tenía constancia de dicho retraso. D’Angiviller se había exiliado por orden del rey el 28 de julio, 14 días después de la toma de la Bastilla. ${ }^{37}$ Desde el exilio seguía manejando la organización del Salón, actuando en su nombre Charles-Étienne-Gabriel Cuvillier como director general de Bellas Artes en funciones. Así, el 10 de agosto, Cuvillier le dirige una carta a Vien, por entonces director de la Academia que, entre otros asuntos, decía lo siguiente:

[...] El Señor Director General piensa que no se debe tener demasiada precaución en la elección de los temas que se expondrán, en relación con las interpretaciones que podrían escapar de un observador y despertarse en otros. [...]. Solo siento aún más lo difícil que es predecir todo lo que se pueda imaginar, y mi objetivo único es instar al comité a que tome todas las precauciones posibles. [...]. Es a este respecto que me consuela, todo lo consolado que pueda estar yo, saber que la pintura del señor David está lejos de estar terminada. ${ }^{38}$

En primer lugar, en esta carta se deja patente el miedo de D'Angiviller a la interpretación de las obras en un clima de levantamiento social como el que estaba viviendo París. Por lo tanto, ya eran conscientes de que había ciertas obras potencialmente subversivas. En cuanto al Bruto, Cuvillier deja notar su esperanza de que no esté acabado para su exposición en este Salón. Así, a través de sus palabras se intuye que para el 10 de agosto de 1789 D’Angiviller

34. DAvid CARRIER: «Was David a Revolutionary Before the Revolution? Recent Political Reading of The Oath of the Horatii and The Lictors Returning to Brutus the Bodies of His Sons», en Dorothy Johnson (ed.): Jacques-Louis David: New Perspectives, University of Delaware Press, Cranbury, 2006, pp. 107-117.

35. Además del Bruto, David expuso otras obras en el Salón de 1789: el Retrato de Antoine Lavoisier y su esposa (1788) y Los amores de Paris y Helena (1788).

36. Robert L. Herbert, David ..., 1972, p. 52.

37. Ibidem, pp. 55-56.

38. TdA de la carta reproducida en Ibidem, pp. 124-125. 
ya conocía la escena planteada por David en su obra y, por consiguiente, conocía y temía sus implicaciones ideológicas.

Dos días más tarde al envío de la misiva, se publicaba un artículo en el periódico radical l'Observateur en el que se culpaba a D'Angiviller y Cuvillier de prohibir la exposición del cuadro de David en nombre del rey. ${ }^{39}$ Cuando Cuvillier negó las acusaciones en este periódico, otro artículo le volvió a contestar diciendo que el día 11 de agosto (al recibir Vien la carta), Vien fue a la casa de David para convencerle de que no entregase el Bruto en el Salón. ${ }^{40}$ Sin embargo, en ese momento David tuvo que confirmarle que lo entregaría, pues Vien, encargado de la edición del catálogo, hizo que imprimiesen el título de la obra de David con una nota indicando su retraso. Según Dowd, el cuadro se exhibió, precisamente, por las presiones de la opinión pública tras haberse sugerido la censura de la obra en el citado artículo periodístico: «[...] popular feeling was so aroused that the 'subversive' picture had to take its appointed place at the Exhibition where it was protected by art students wearing the uniform of the newly created National Guard». ${ }^{41}$ Lo cierto es que, justo al terminar el cuadro, la actitud intransigente de David se vería reafirmada en un acto de rebeldía: el mismo día de la exposición del Bruto (12 de septiembre), David se unió a las demandas de un grupo de jóvenes artistas contra la Academia, llegando a liderar esta oposición. ${ }^{42}$ En esta rebelión creciente de David pudo haber también cierto acto de venganza, pues en 1787 D'Angiviller le había negado al pintor el puesto de director de la Academia de Francia en Roma, alegando que era demasiado joven..$^{43}$ Además, también en septiembre de 1789, la mujer de David se presentó como parte de una delegación de mujeres artistas (y esposas de artistas) en la Asamblea Constituyente para donar sus joyas a la causa del pueblo francés ${ }^{44}$ recreando así una leyenda romana relativa a la donación de joyas a la República que había sido llevada al lienzo, precisamente, en el Salón de 1785 por Nicolas-Guy Brenet. ${ }^{45}$

En cuanto al resto de obras que se expusieron en el Salón de 1789, destaca cierto eclecticismo ideológico: desde retratos del rey Luis XVI como el de Antoine-François Callet hasta las propias escenas de la toma de la Bastilla de Hubert Robert. También se colgaban cuadros de tono amable, como el Amor escapando de la esclavitud, de Joseph-Marie Vien, y escenas bíblicas

39. Robert L. Herbert, David ..., 1972, p. 62.

40. Ibidem, pp. 62-63.

41. David Lloyd Dowd: Pageant-Master of the Republic. Jacques-Louis David and the French Revolution, University of Nebraska Press, Nueva York, 1948, p. 19.

42. Robert L. Herbert, David ..., p. 52.

43. Thomas Crow, Painters and ..., p. 234.

44. Antoine Schnapper, David, 1982, p. 97

45. Robert L. Herbert, David ..., p. 52. 
como El diluvio, de Jean-Baptiste Regnault. El día de la inauguración, cuando el Bruto aún no estaba en la pared, un artículo en Révolutions decía:

En efecto, las alegorías del amor, los retratos de cortesanas y los halagos de los esclavos nos interesan muy poco. De ahora en adelante, Bruto, pronunciando la muerte de sus hijos, o Decio muriendo por su patria, eso es lo que nos complacerá y nos seducirá. ${ }^{46}$

De este modo, era visible el agotamiento de la temática cortesana y la predisposición a descubrir nuevas historias que unieran al pueblo en un sentimiento común.

\section{La intención propagandística de la monarquía y la intención estética de David}

El Bruto expuesto en el Salón de 1789 deja patentes dos intenciones diferentes en la concepción y creación de la obra: la intención propagandística de la monarquía y la intención estética de David. En un primer término, la monarquía tiene la intención de afirmar su poder mediante el encargo de un cuadro de pintura de historia en el cual, siguiendo el programa propuesto por d'Angivillier, se muestre una virtud patriótica que beneficie a la imagen del rey Luis XVI. Y, en un segundo término, David, al recibir el encargo, tiene la intención estética de innovar, no a través del formato estético (pues el estilo neoclásico ya se había consolidado en aquella fecha), sino a través de la forma de representación. Su idea de innovar en el modo en que los personajes conforman la escena, creando un episodio ausente hasta entonces en las referencias culturales del público, queda patente en la carta que dirige a su alumno Wicar, referida anteriormente. De hecho, en otra carta escrita cuando el cuadro se colgó en el Salón, David le comenta a Wicar que el Bruto fue cubierto de alabanzas: «Elogian principalmente la concepción y especialmente el colocarlo [a Bruto] en la sombra». ${ }^{47}$ Es decir, esta intención estética fue rápidamente reconocida.

Los datos extraídos del contexto de creación de la obra confirman que, dado el amplio margen de creación artística que el encargo real le otorgaba a David, el artista contó con espacio suficiente dentro de la obra para configurar el significado del mensaje. David eligió (1) el tema histórico, (2) el momento exacto de la historia y (3) su representación, lo cual no desvirtuaba en principio la intención preliminar de la monarquía: pintar un cuadro de

46. TdA de la cita en Antoine Schnapper, 1982, p. 65.

47. TdA de la carta citada en Robert L. Herbert, David..., Allen Lane, Londres, 1972, p. 124. 
historia que comunique una virtud patriótica. Así pues, la intención de poder de la monarquía queda explícita en esta obra principalmente porque se expone en un salón auspiciado por el rey, y porque se nombra en un catálogo donde se indica que el Bruto es un encargo real. La interpretación en este contexto, por consiguiente, es inequívoca. Pero, una vez finalizado el Salón, la innovación estética de David acabaría apuntando hacia la necesidad de terminar con la monarquía por amor a la patria.

\section{El contenido ideológico y estético del Bruto}

Las características del Bruto son especiales, ya que David contó con una libertad creativa tal que la obra representa, en términos generales, la transición entre el trabajo del artista para las instituciones oficiales y la independencia ideológica del mismo.

La Casa Real pretendía legitimar su poder a través de la idea de patriotismo de la obra. Ese patriotismo es una virtud que posee el héroe de la escena: Bruto, quien debe concentrar, como en toda pintura de historia realizada para Luis XVI, unos valores extrapolables a la monarquía. No obstante, el Bruto se puede leer en otros términos como obra de transición, puesto que, en el contexto en que se está estudiando, la Revolución Francesa recorría las calles y los acontecimientos circundantes favorecieron la reinterpretación de la obra. En este sentido, Bruto sigue siendo el héroe de la escena, pero transmitiendo un mensaje mucho más literal al público: la defensa de los ideales y la lucha por la nación frente a la monarquía tiránica. En la historia original, Bruto había llegado al punto de condenar a muerte a sus propios hijos, no solo por la defensa de un ideal abstracto de la nación, sino por la lucha a muerte contra la monarquía.

De este modo, el sentido que pareció alzarse como definitivo queda patente en el propio desarrollo de la intención estética del artista. Mientras que la pintura de historia solía representar episodios en los que las hazañas heroicas se situaban en la vía pública y se rodeaban de testigos, en el Bruto (como ya hizo David por primera vez cuatro años antes con los Horacios) la acción sucede en la intimidad del hogar. Aquí, el público receptor se asoma a la privacidad del personaje, lo que le permite al artista transmitir las pasiones con más fuerza. Pero, además de innovar en el escenario, David lo hace también en la narración, representando un momento de máxima angustia que no había llegado a aparecer en las fuentes históricas. La acción de la narración se ubica realmente en un segundo plano, donde los lictores están irrumpiendo en la casa del cónsul romano para dejarle los cuerpos sin vida de sus hijos, de los cuales, como espectadores, solo podemos ver unas piernas 
iluminadas (figura 2). Este regreso de los hijos como cadáveres provoca una reacción contenida en Bruto, que sí está en un primer plano justo delante de sus hijos, aunque sombrío. La iluminación del fondo, que marca el detonante de la obra, en contraste con un protagonista oscuro, no hace sino acrecentar la tensión otorgada a Bruto. Cuando la vista se centra en él, hay ciertos elementos que transmiten su tormento: su ceño fruncido, sus pies cruzados con los dedos encogidos (única parte de Bruto iluminada) y su mano izquierda arrugando un papel escrito. Este papel es, según Herbert, una carta que le confirmaba la traición cometida por sus hijos. ${ }^{48}$ Mientras lo agarra con fuerza, se apoya en los símbolos que le han dado la autoridad y el espíritu para cometer tal acción: son las ideas de Roma (en la loba capitolina del pedestal) y de la justicia (en Minerva, la diosa de la sabiduría y las causas justas). Bruto ha matado a sus hijos, conspiradores para la restauración monárquica, por el bien de Roma. La postura de Bruto y, concretamente, su mano derecha, indica que acaba de ser interrumpido mientras hundía la cabeza entre las manos. Ahora afronta una realidad para la que debe mostrar entereza, teniendo que ratificar la supremacía de su rol político al rol familiar. Aquí es donde se manifiesta en su máxima expresión el patriotismo pretendido por la monarquía como virtud ejemplar. Esta responsabilidad en Bruto queda patente en la mirada fija que dirige hacia el público, elemento crucial. En cierto modo, lo está interpelando, esperando a ser juzgado por tal atrocidad.

Estos acontecimientos se comprenden una vez que la mirada del espectador ha sido guiada hasta ahí a través de los llantos de las mujeres de la familia. Ese es el punto focal de la obra, donde recae toda la iluminación (figura 3). La mujer y las hijas de Bruto son las que realmente reciben los cadáveres, pues se sitúan frente a ellos, por lo que, como el propio David le indicaba a Wicar en su carta, es el lamento de las mujeres lo que ha interrumpido la aflicción en la que se encontraba Bruto. Ellas también han sido interrumpidas mientras realizaban labores domésticas (acción contenida en el costurero encima de la mesa), por lo que quizá aún no habían sido informadas de lo que estaba a punto de ocurrir. Este hecho aumenta aún más el patetismo en sus reacciones. En ellas vemos distintos grados de sufrimiento: la madre extiende el brazo hacia sus hijos mientras tiene que darle apoyo a su otra hija, desmayada del dolor. Bajo su pecho también se encuentra una hija que intenta ocultar de sus ojos los cadáveres de sus hermanos. La criada, por otro lado, oculta completamente su rostro. La madre de la familia, eje y personaje principal de la composición, es por tanto la única que lidia de manera directa con su dolor reflejando, a la vez, el papel de protectora del que Bruto ya se ha desprendido como padre.

48. Robert L. Herbert, David ..., p. 128. 


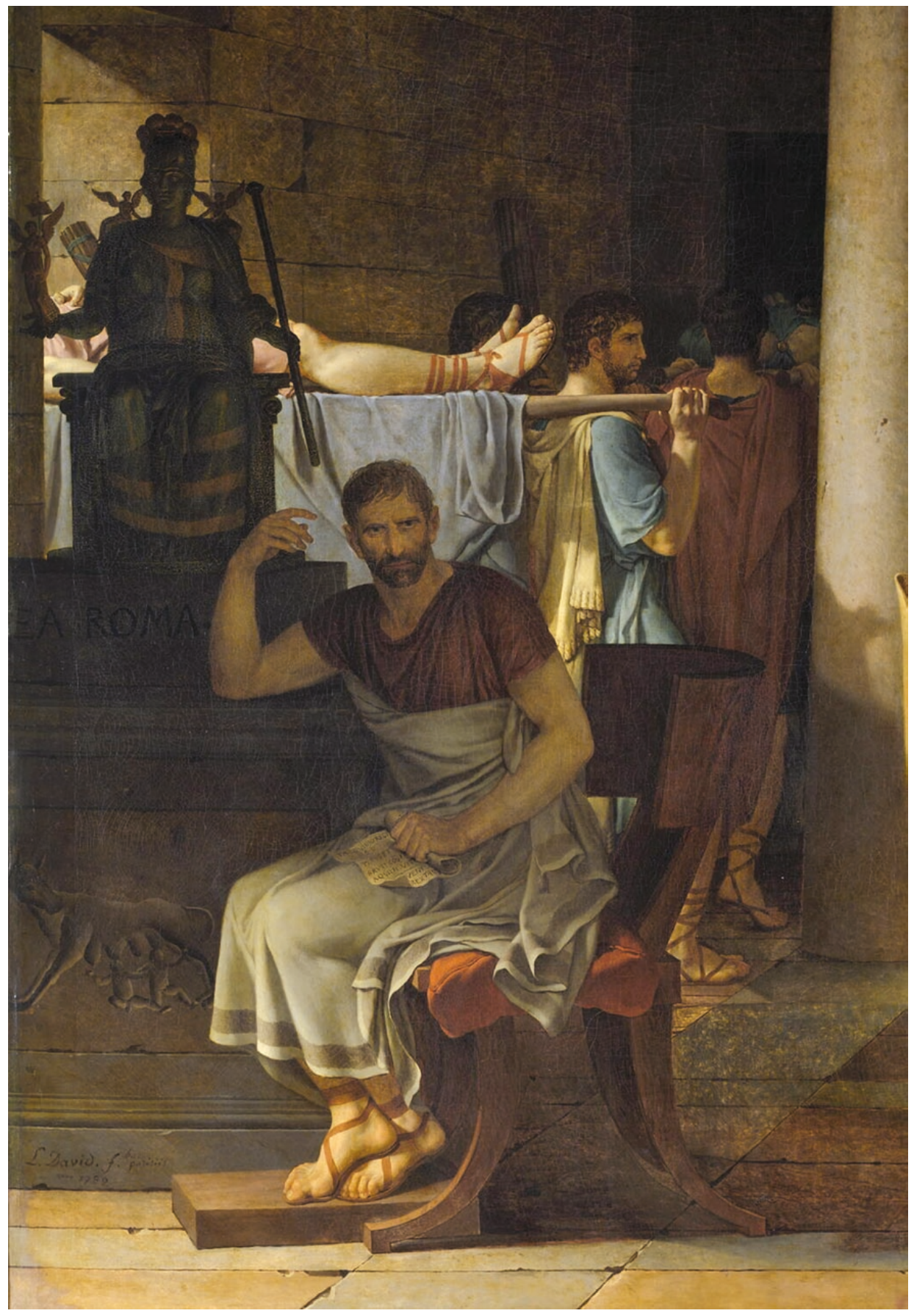

Figura 2: Detalle de Bruto en Los lictores devuelven a Bruto los cadáveres de sus hijos (Jacques-Louis David, 1789) 


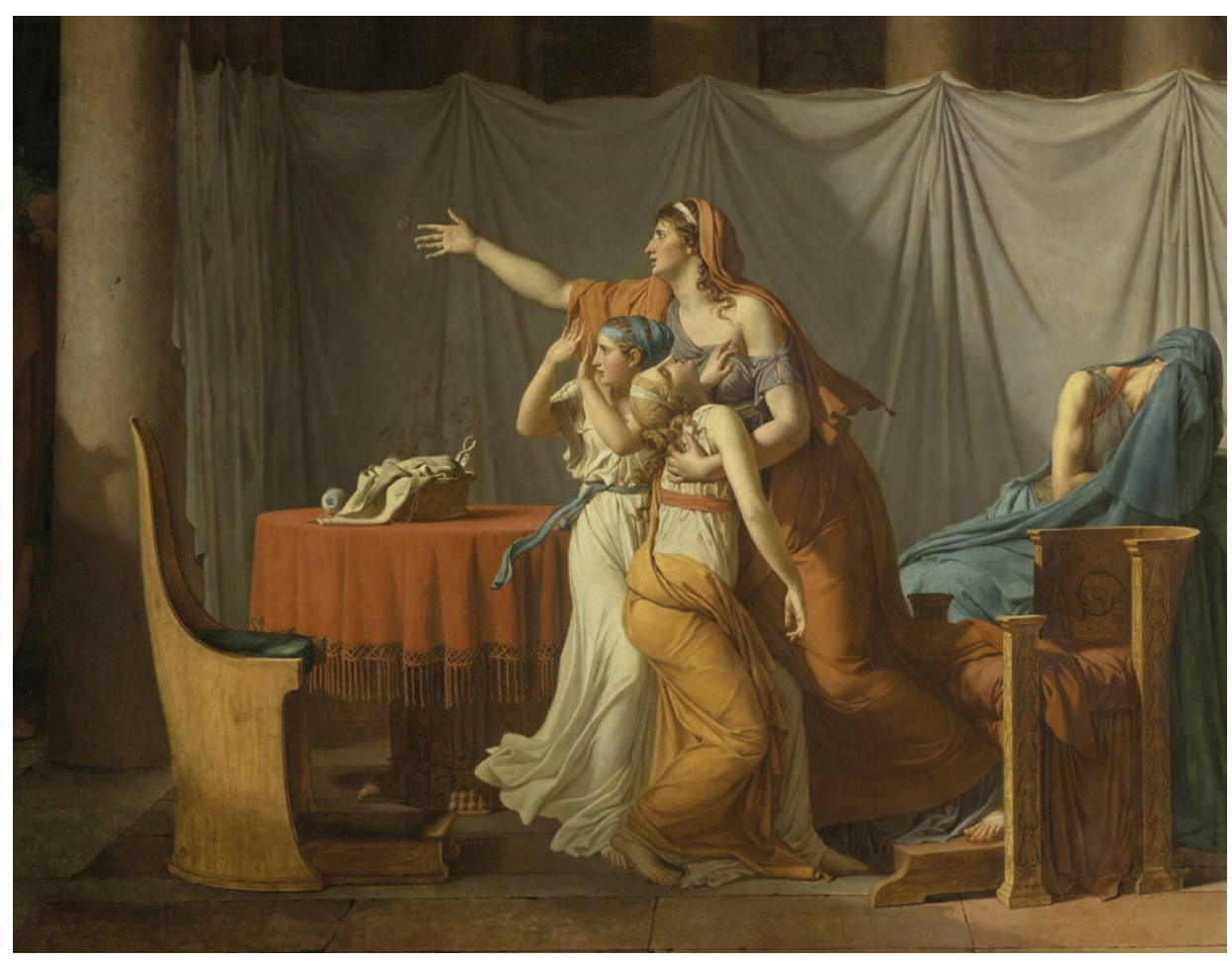

Figura 3: Detalle de las mujeres de la familia de Bruto en Los lictores devuelven a Bruto los cadáveres de sus hijos (Jacques-Louis David, 1789)

\section{Instrumentalización propagandística del Bruto en el Salón de 1789}

Puesto que el encargo que recibió David no fue restrictivo, las técnicas de instrumentalización propagandística en relación al contenido la obra fueron mínimas. De hecho, al dejar la elección del tema en manos de David, no se llegó a establecer la presencia de ningún tipo de contenido. En última instancia, el único que puede identificarse como tal es el género histórico, con el que ya se había educado la mirada del público para que lo relacionase directamente con el programa iconográfico real. Sin embargo, en las técnicas de formato podemos mencionar las dimensiones de la obra, pues al ser un encargo estas debían quedar acordadas por contrato, ya que en función de las mismas el artista recibía una mayor o menor cantidad de dinero. El cuadro de Bruto tiene 323 $\mathrm{cm}$ de alto por $422 \mathrm{~cm}$ de ancho, algo ligeramente inferior a las medidas de su anterior pintura de historia (los Horacios, de $330 \times 425 \mathrm{~cm}$ ). Con estas obras de gran tamaño se pretendía, ciertamente, impresionar al público visitante.

Por otro lado, en cuanto a las técnicas contextuales relativas a la exposición en el Salón de 1789, encontramos el libreto como herramienta 
clave propagandística. Quizá lo más relevante de él en cuanto a la obra es su descripción: Bruto, primer Cónsul, de vuelta en su casa, después de haber condenado a sus dos hijos, que habian conspirado contra la libertad romana, los lictores devuelven sus cuerpos para que sean enterrados. ${ }^{49}$ Así pues, ocultando el hecho de que los hijos habían conspirado para la restauración monárquica, Bruto se alza según el libreto como un héroe de la libertad, no como un héroe republicano como se interpretaría pocos años más tarde. Además, como en Salones anteriores, en el libreto se redundaba acerca del poder de la monarquía y su voluntad de llevar la exposición a cabo. Otra de las técnicas contextuales relevantes es la disposición de las obras dentro del entorno expositivo. En el caso del Salón de 1789, podemos acudir al grabado realizado por Charles de Wailly, en el que se ha marcado con un recuadro en rojo el lugar que ocupaba el Bruto. ${ }^{50}$ En este grabado se observa claramente (además del recargamiento de las paredes del Salón) la regla expositiva que se estaba siguiendo: colocar las obras más pequeñas más cerca de la vista del público, y las más grandes en los huecos más altos. Por tanto, esta obra de David no se situaba a la altura de los ojos del público, sino en el espacio reservado para los lienzos de historia, pues eran habitualmente los de mayor dimensión. No obstante, en el grabado de De Wailly se puede apreciar que, de las 350 obras del Salón, ${ }^{51}$ el Bruto ocupaba una posición privilegiada, ya que, colocado en el muro frontal, se podía apreciar desde la planta baja, esto es, desde la perspectiva adquirida en el grabado. No ocurre así, por ejemplo, con las pinturas de los muros laterales, que solo pueden observarse correctamente desde el espacio reducido de la primera planta. Se potenciaba de este modo el impacto que pudiera generar la obra en el público.

\section{EL BRUTO DESPUÉS DEL SALÓN DE 1789}

El Bruto de David fue, quizá, su obra más simbólica en tanto que convirtió a Bruto en el héroe republicano de la Revolución. Por lo que supuso la introducción de este personaje en la vida francesa del cambio de siglo, se podría afirmar que la monarquía no logró el objetivo de reafirmar su poder a través de esta obra de propagada, ni de revestirse de la virtud patriótica que destilaba la obra. Todo lo contrario: no hubo nada en este lienzo que fortaleciese

49. Durning Lawrence EtTlinger, «Jacques Louis David ...», p. 117.

50. https://sites.google.com/a/plu.edu/paris-salon-exhibitions-1667-1880/salon-de-1789.

51. YURI LONG: «An evolving document: growth and development of the Salon Livret», en JoHN Hagood: Documenting the Salon: Paris Salon Catalogs, 1673-1945, National Gallery of Art Library, Washington, 2016, p. 71. 
el papel de Luis XVI en la mente del público. Este Salón en concreto tiene la particularidad de ser el primero abierto una vez había estallado la Revolución, y el último organizado por la Casa Real. Conociendo de antemano el levantamiento del Tercer Estado, se puede intuir que la actitud del pueblo a la hora de interpretar los cuadros colgados en este Salón debía ser distinta a la de los eventos anteriores: había evolucionado desde una receptividad pasiva a su verdadera formación como público crítico y activo. De las obras esperaban, pues, que diesen una respuesta estética a sus necesidades cívicas; que, en definitiva, pudieran verse representados en ellas de algún modo.

No obstante, las críticas recogidas del Salón de 1789 describían el cuadro en su plano estético, sin llegar a realizar una valoración ideológica de la obra. La gran mayoría, y tal y como David le avanzaba a Wicar, elogiaba la creación del momento dramático, la innovación de la composición y, con ella, el hecho de ubicar al protagonista en la sombra para intensificar su duelo. El 17 de septiembre se leía en Affiches:

M. David, cuyo nombre es suficiente para atraer la atención de los conocedores, se lleva todos los honores nuevamente este año. Su Bruto... es una composición de un tipo absolutamente nuevo, uno cuyo estilo es noble, severo, enérgico, y que retrata de la manera más adecuada una escena tan conmovedora como aterradora. La idea de poner a Bruto completamente en la sombra es un golpe de genio, que ayuda a hacer que la figura sea siniestra y desencadena el interesante grupo formado por la madre y las hermanas de las desafortunadas víctimas de la severidad paterna. ${ }^{52}$

Por otro lado, las críticas más institucionales sí parecían adentrarse en el significado del cuadro para reafirmar su concepción original como encargo real. Así, en el Supplément aux Remarques sur les ouvrages exposés au Salon, el Conde de Mende Maupas oscurecía el incipiente republicanismo de la obra del siguiente modo:

Para apreciar las bellezas sublimes de esta composición, uno debe volver a la época en que Roma construyó su libertad sobre la tosquedad de sus costumbres, cuando los aspirantes a ciudadanos solo destronaron a reyes para reinar, cuando los sentimientos naturales dieron paso a la ambición ardiente, cuando un fantasma republicano consoló al pueblo por la tiranía de sus cónsules. Entonces uno comprenderá los méritos de la pintura de M. David: fuerza de composición, nobleza de expresión, decisión de movimiento, agonía de pose y, más que todo eso, originalidad de la concepción, porque el tema principal se encuentra en la parte oscura de la obra, como

52. TdA de la crítica citada en Robert L. Herbert, David ..., p. 126. 
para marcar el sufrimiento de un ser a quien la morgue republicana no puede evitar ser padre..$^{53}$

La república romana es asociada aquí con un concepto retrógrado y vago que, bajo la premisa de acabar con la tiranía, se apoderaba de la ambición de los ciudadanos y los acababa haciendo sufrir hasta el nivel más íntimo que refleja Bruto. Chua afirma que esta crítica se acerca a un ataque hacia el sistema republicano en general y su imposibilidad de llevarse a cabo $^{54}$; no obstante, la descripción ideológica de la obra no es directa, o, al menos, no tan directa como llegó a ser al siguiente año.

Habría que esperar algunos meses, ya en 1790, para que tuviese lugar un evento en el que la obra de David adquirió un significado republicano y tuvo un efecto directo en el comportamiento del público. Se trata de la representación de Bruto de Voltaire el 17 y el 19 de noviembre de 1790. Anticipándose a los posibles altercados que podía ocasionar este regreso de la tragedia de Bruto en un contexto revolucionario, París prohibió el día del reestreno que la audiencia llevase espadas al teatro. En la sala había quienes apoyaban al rey, y aplaudían las líneas de Bruto en que defendía la causa de Roma, mientras que los radicales se exaltaban en los momentos en que Bruto se enfrentaba a la tiranía monárquica. Tal era el entusiasmo que, al final de la obra, los radicales pidieron que se trajese el busto de Voltaire y lo colocaron en el escenario para coronarlo con laurel. ${ }^{55}$ En la siguiente representación los defensores de la monarquía se redujeron en número, intimidados por los vítores de los radicales. ${ }^{56}$ La noche del 19, cuando se levantó la cortina, el público pudo ver a un lado el busto de Voltaire y al otro el busto de Bruto, el cual había traído David desde Roma. ${ }^{57}$ Sin embargo, lo más relevante ocurrió al final de la obra, cuando en la última escena se recreó el cuadro de David, inmediatamente reconocido por el público: «Every Parisian knew David's painting; each recognized right away the intention of thus honoring the artist in public in front of the nation. It was like a national holiday, heightened by unanimous applause». ${ }^{58}$

En efecto, los eventos del año siguiente no hicieron más que acentuar la estrecha relación entre Bruto, el republicanismo en Francia, y David. Cuando en junio de 1791 se interceptó a Luis XVI intentando salir del país, el Club de los Cordeliers (o Sociedad de Amigos de los Derechos del Hombre y del Ciudadano) colgó un cartel en la ciudad en el que había cambiado de los

\footnotetext{
53. Ibidem, p. 127.

54. Kevin ChuA: «In the Shadow of David's Brutus», en Representations, 21(1), 2013, p. 126.

55. Robert L. Herbert, David ..., pp. 74-75.

56. Ibidem, pp. p. 15.

57. Ibidem, pp. p. 76.

58. Von Halem citado en Robert L. Herbert, David ..., 1972, pp. 77-78.
} 
versos de Voltaire las referencias a Tarquinio y Roma por referencias al rey y Francia. ${ }^{59}$ Además, el mes siguiente David organizó el funeral de Voltaire, siendo uno de los eventos más destacados que realizó como maestro de ceremonias. ${ }^{60}$

Por último, el momento que supuso la resemantización completa del Bruto de fue su exposición en el Salón de 1791. En este evento se presentó por primera vez el boceto de David para el Juramento del Juego de Pelota, y con el objetivo de reforzar el compromiso republicano del artista, la Asamblea Nacional (organizadora del Salón) permitió que David expusiese de nuevo a los Horacios y al Bruto, produciéndose así una transferencia de significado desde el encargo jacobino (el Juego de Pelota) hacia los dos lienzos de historia originalmente concebidos como propaganda monárquica. De hecho, al Bruto se le modificó el título por Brutus, de retour chez lui, après avoir condamné ses deux Fils qui sétoient unis aux Tarquins, dont on rapporte les corps pour leur donner la sépulture ${ }^{61}$. Si el en Salón de 1789 se indicaba en el libreto que los hijos de Bruto habían conspirado «contra la libertad romana», aquí se deja claro que estaban aliados con los reyes Tarquinos, por lo que el sentido anti-monárquico de la obra cobra fuerza en 1791.

La figura de Bruto y su amor por la república se siguió recordando en los años posteriores, ya que se llegó a colocar un busto del cónsul delante de los portavoces de la Convención Nacional. ${ }^{62}$ Pasó a ser, en definitiva, el héroe y referente republicano por excelencia. De hecho, cuando acusaron a ya un reconocido republicano David de haber aceptado retratar en 1792 al rey Luis XVI enseñándole la constitución a su hijo (encargo que, ciertamente, comenzó), ${ }^{63}$ el pintor respondió: «iLes diré que el pintor de Bruto no se hizo para pintar reyes!». ${ }^{64}$ En palabras de Lee, «as the Revolution proceeded David's picture acquired a topicality that he could not have foreseen and its dreadful subject and memorable image entered the public's consciousness». ${ }^{65}$

\footnotetext{
59. Robert L. Herbert, David ..., p. 81.

60. Dowd, 1948, p. 46

61. Sin Autor: Explication des peintures, sculptures, et gravures, de messieurs de l'Académie Royale, Imprimerie des Bâtimens du Roi, París, 1789, p. 23.

62. Robert L. Herbert, David ..., p. 16.

63. Antonio Pinelli, David, 2004, p. 21.

64. TdA de David citado en Antoine Schnapper, David, p. 121.

65. Simon LeE, David, pp. 126-127.
} 


\section{BIBLIOGRAFÍA}

Boime, Albert: «Marmontel's Belisaire and the pre-revolutionary progressivism of David», en Art History, 3(1), 1980, pp. 81-101.

- Historia social del arte moderno. 1. El arte en la época de la Revolución, 1750-1800, Alianza Forma, Madrid, 1994 (1987).

Brookner, Anita: Jacques-Louis David, Purnell, Paulton, 1980.

CARrier David: «Was David a Revolutionary Before the Revolution? Recent Political Reading of The Oath of the Horatii and The Lictors Returning to Brutus the Bodies of His Sons», en Dorothy Johnson (ed.): Jacques-Louis David: New Perspectives, University of Delaware Press, Cranbury, 2006, pp. 107-117.

ChuA, Kevin: «In the Shadow of David's Brutus», en Representations, 21(1), 2013, pp. 107139.

Crow, Thомаs: Painters and Public Life in Eighteenth Century Paris, Yale University Press, New Haven, 2000.

Dowd, David Lloyd: Pageant-Master of the Republic. Jacques-Louis David and the French Revolution, University of Nebraska Press, Nueva York, 1948.

Etthinger, Durning Lawrence: «Jacques Louis David and Roman Virtue», en Journal of the Royal Society of Arts, 115(5126), 1967, pp. 105-123.

Germer, Stefan y Kohle, Hubertus: «From the theatrical to the aesthetic hero: on the privatization of the idea of virtue in David's Brutus and Sabines», en Art History, 9(2), 1986, pp. 168-184.

Herbert, Robert L.: David Brutus, Allen Lane, Londres, 1972.

Honour, Hugh: Neo-Classicism, Penguin Books, Victoria, 1968.

LEE, Simon: David, Phaidon, Londres, 1999.

LONG, YURI: «An evolving document: growth and development of the Salon Livret», en Hagood, John: Documenting the Salon: Paris Salon Catalogs, 1673-1945, National Gallery of Art Library, Washington, 2016

Pinelli, Antonio: David, 5 Continents, Milán, 2004.

Prieto, Fernando: La Revolución Francesa, Itsmo Madrid, 1989.

Schnapper, Antoine: David, New York Alpine Fine Arts Collection, Nueva York, 1982.

Sin Autor: Explication des peintures, sculptures, et gravures, de messieurs de l'Académie Royale, Imprimerie des Bâtimens du Roi, París, 1789. Recuperado de: gallica.bnf.fr/.

White, James Llewelyn: The Origins of Modern Europe, Horizon Press Publishers, Nueva York, 1965. 